\title{
The Identical Data in Cloud Storage with ADJDUP Technique
}

\author{
Anubhav Pandit \\ Department of Computer Science and Engineering, \\ Sachdeva Institute of Technology, Mathura (SIT Mathura), \\ Dr. A P J Abdul Kalam Technical University, \\ Lucknow, Uttar Pradesh, \\ India.
}

\begin{abstract}
Data deduplication is necessary for corporations to minimize the hidden charges linked with backing up their data using Public Cloud platform. Incapable data storage on its own can become improvident, and such problem are enlarging in the Public Cloud at other and scattered satisfied confirmed storage structure are creating multiple clone of single account for collating or other purposes. Deduplication is friendly in cost shrinking by lengthening the benefit of a precise volume of data. Miserably, data duplicity having several safety constraints, so more than one encoding is appropriate to validate the details.
\end{abstract}

There is a system for dynamic Information-Locking and Encoding with Convergent Encoding. In this InformationLocking and Encoding with Convergent Encoding, the data is coded first and then the cipher text is encoded once more. Chunk volume is used for deduplication to diminish disk capacity. The same segments would still be encrypted in the same cipher message. The format of the key neither be abbreviated from encrypted chunk data by the hacker. The comprehension is also guarded from the cloud server. The center of attention of this document is to reducing disk storage and provides protection for online cloud deduplication.

Keywords: data compression; information systems; cloud computing; encryption; secure storage;

\section{INTRODUCTION}

Data deduplication is a procedure for removing unnecessary information from the complete information source. In the process of deduplication, extra copies of the same information are removed, providing only one copy to be stored. Information is analyzed to recognize duplicate byte patterns to ensure the single instance is indeed the single file. Then, replicated data are replaced with a reference that points to the stored chunk, performing data deduplication before moving data to the Cloud to turn down Cloud price and avoid undesirable shock.

Now Days, as cloud inherited by the companies and they are shifting towards data storage on distinct cloud environment, data deduplication performs a more crucial role rather than simply saving on storage costs. In merger with cloud-based object storage architecture, efficient data deduplication is opening up new opportunities to do more with stored data. Data deduplication refers to a technique for eliminating redundant data in a data set. In the process of deduplication, extra copies of the same data are removed, leaving only one copy to be stored. Data is analyzed to identify duplicate byte patterns to ensure the single instance is indeed the single file. Then, duplicates are replaced with a reference that points to the stored chunk.

Thus, deduplication will greatly reduce the space used to hold the huge data collection. Data protection and privacy is another aspect of growing interest in advanced data systems, but deduplication and encryption are controversial. Deduplications make advantage of knowledge similarities to obtain a deduction in size. Whereas the necessity of cryptography for creating cipher content which is clone from theoretical uncommon content. The purpose of a secure deduplication environment is to supply data protection, from the two within and external foe, with the space capacity that can be gained by single occurrence storage system. We are interpretating dual methods for working and confirm stable deduplication. All though all forms are comparable, which provide rather extraordinary safety illuminations.

ISSN: 2582-337X

Submitted: 04.12 .2020

Accepted: 30.12.2020

Published: 06.01.2021

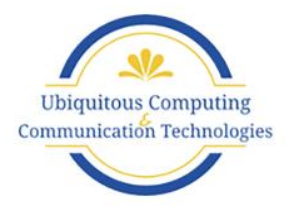


Journal of Ubiquitous Computing and Communication Technologies (UCCT) (2020)

Vol.02/ No.04

Pages: 214-218

https://www.irojournals.com/jucct/

DOI: https://doi.org/10.36548/jucct.2020.4.004

Both may be extended to the administrate of a single server without the creation of a circulated space. In the first oneserver storage, user transmit with a single record server that grasps all details and metadata. Although, metadata is disposed on an independent metadata site, and data are disposed on the improvement of object-dependent calculating appliances.

The two versions for secure deduplication method rely upon multiple of simple secure structures. The first instance utilizes position-based information which encourage encoding while allowing standard partition to be deduplicated. Joined encryption requires a plain text feature as an encryption key: any user's decoding a specified piece can use the similar key for the same, similar identical plain text would encode identical content, paying no appraise to who scrambles them. Whereas this method advice that a specific cipher text occurs as of today, in these sections, plain text. Foe should not infer the key from the scrabbled chunk without some plaintext knowledge. Second, both content lumping and encryption lives on client details, plain text copy is occasionally swapped, and the system is reinforced in opposition to the two inner and outer foes (1). Finally, the instruction that partners are part of a specified record is secured with a noteworthy key, monitoring the result of a main event to a single text (2). In contrast, the keys are set within the environment in such a move that clients literally needed to hold a sole private key with no observation to the number of records of which they are confirmed.

\section{LITERATURE REVIEW}

Cloud storage is one of the latest technologies; the key objective is to grant the optimal employment of data center services (3). Replica files may be studied in two ways, one on the server side and the other on the client side. The server-side replicate until the file has been submitted to the internet, however the client-side replicate until uploading the document customer tests the physically utilize the hash key for dispatching functionality (4). Key benefit of deduplication is that it diminishes volume and enlarge performance (Y. Yang, H. Zhu (2017). The degree of deduplication that can be done is counted by the number of solicitudes. Modern market histories, deduplication figures varying from 4:1 (75 per cent) to 500:1 (99.8 per cent) are similar. While deduplication helps storage suppliers, it also puts users at risk of privacy. unplanned threshold form used for brute-force attacks. Customer and server-side deduplication framework (6) may be settled. personal deliberation is a problem among caching of cloud information; privacy deduplication formation is based upon classic encryption scheme (7). Multiple, collaborative Message-Locked Encryption (IMLE) and Convergent encryption technique. Convergent technique of encryption having the hash key material. The cryptographic algorithm manufactures same chipper content from the same plain text. This is one device form used in cloud storage for the removal of duplicate files (8). The Client encrypts its plain text $\mathrm{V}$ in a $\mathrm{B}$ format that is itself a determinist hash of the plain text $\mathrm{m}$ with a deterministic, collaborative Message-Locked Encryption (IMLE). The algorithm A, P, R, S of any Message-Locked Encryption (MLE) method. MLE will provide protection only for unreliable data inside this two-dimensional context $(9,10)$. Association and parameter dependency are two ways of protection for MLE one. Association implies the reliability of the message is connected to the other parameter dependency as it is encrypted and unconventionally unstable. The encryption often extends to messages based on the public parameter. IMLE is fascinating in its own files and provides a range of supplementary convenience are provided with first stable deductibility plans which enable growing updates.

Collision is the similar hash value with multiple separate information bring about. Information storage creates a hash collision (11) as data duplicity happens. The biggest downside to deduplication is the capacity surplus. This is a big machine concern that often impacts machine systems that implementations. In this case the overhead device associated with the computation of hash vales is a crucial part of the deduplication process.

\section{Goal Vs Deduplication Source}

Another way is that it appears to advice knowledge deduplication. The information can be checked level by level and deduplication of information spontaneously detected. It is mentioned to be as deduplication goal. Origin deduplication allows deduplication of knowledge about the source of information. This frequently occurs specifically inside a text setting. On times, the data system can analyze fresh documentation and equate them with the risks of current data. The purpose is to remove duplicity from a backup contact, when it moves across a request between the source and the reserves reason.

ISSN: 2582-337X

Submitted: 04.12.2020

Accepted: 30.12.2020

Published: 06.01.2021 
Journal of Ubiquitous Computing and Communication Technologies (UCCT) (2020)

Vol.02/ No.04

Pages: 214-218

https://www.irojournals.com/jucct/

DOI: https://doi.org/10.36548/jucct.2020.4.004

\section{SYSTEM ANALYSIS}

\section{Existing system}

Distributed computing exceptional motivates data providers which required the externalization of their data to the cloud unknowingly exposing their elegant data to outside collections and may require customers with specific authorization to get the data. This contemplate data should be placed in encrypted figure with obtain to authorizing methodologies to a level which no one aside from customers with traits (certifications) of specific figure can hamper the scrambled data. An encoded system that encounters this requirement is known as possession-based encryption (ABE). Be that as it may, the standard ABE framework rejects to achieve secure deduplication, which is a procedure to spare storage room and system transmission volume by cleaning out repetitive duplicates of the scrambled information put away in the cloud.

\section{Disadvantages}

1. Data will be encrypted before it is stored in the cloud so that for decoding and data cast about tedious will be there. 2. For the decryption and analysis some procedure required which builds the cost.

3. Now and again it isn't capable for the security of the information.

\section{Proposed system}

We introduced a novel way to accord with understand a trait-based capacity framework carrying safe deduplication. Our capacity framework is worked under blend cloud engineering, where a private cloud manages the calculation and an open cloud deals with the capacity. The private cloud is given a trapdoor key related with the comparing cipher text, with which it can exchange the cipher text more than one access compromise into cipher texts of the same plain text under some other access strategies without observing the hidden plaintext. In the wake of accepting a capacity ask for, the private cloud first checks the permissible of the transferred thing through the included verification.

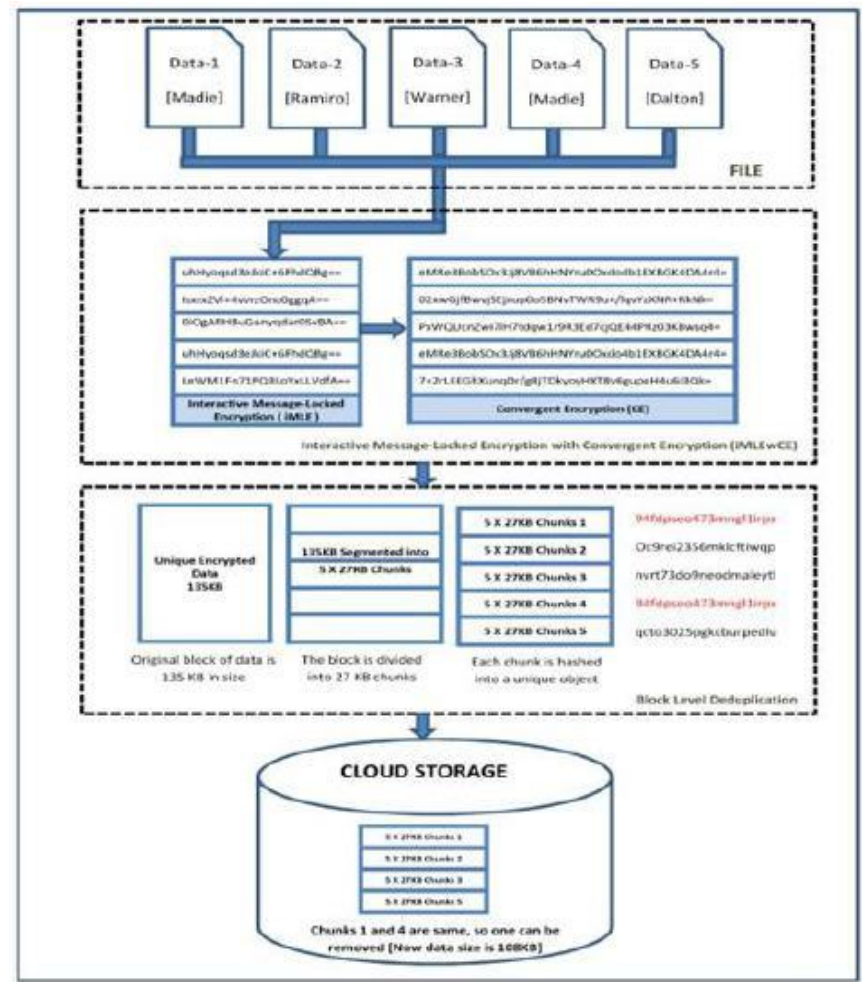

Fig 1: Specify the proposed System where a private cloud manages the calculation and an open cloud deals with the Advantages capacity.

1. It gives cross breed cloud engineering which deals with the space so storage room will be backup.

ISSN: 2582-337X

Submitted: 04.12.2020

Accepted: 30.12.2020

Published: 06.01.2021 
Journal of Ubiquitous Computing and Communication Technologies (UCCT) (2020)

Vol.02/ No.04

Pages: 214-218

https://www.irojournals.com/jucct/

DOI: https://doi.org/10.36548/jucct.2020.4.004

2. Enhanced security.

Modules

The modules are as follows

- Input Detail Source

- $\quad$ Feature Rules

- Cloud

- Users

Input Detail Source

An information supplier needs to outsource his/her information to the cloud and offer it with clients having certain certifications.

\section{Feature Rules}

Feature Rules matters every user a decryption key associated with corresponding set of data.

\section{Cloud:}

The cloud contains open cloud that is answerable for data stockpiling and a private cloud is responsible for making computations, an instance of it is tag checking. while publishing a data stockpiling require, every datum provider right off the bat made a tag $\mathrm{L}$ and mark $\mathrm{L}$ integrated with the knowledge prior to it scramble the knowledge below an existing shape and on top of it an adjustment of properties. Same way, every datum provider makes a proof pf on the association of the name L, stamp P and the blend the messages, however this declaration will not be safeguard wherever in the cloud and is simply used during the validating stage for any as of late transferred limit inquire. Ensuing to getting a limit demanded, the private cloud initially verify the authenticity for affirmation pf, after that checks adjust of the latest name with existing names in the Existing structure. If no new partner lives for this name L, the private cloud incorporates name L other stamp P to a label--name broken-down, and forward name with encoded data, (P, ct) to people in general cloud for volume.

\section{CONCLUSION}

Attribute-based encryption (ABE) having extensive utilizes in cloud computation and information provider provides their encoded information to the cloud and provide information with clients fixed secured information. Whereas at other side, deduplication is a very necessary methodology to acquire distance for applying away and arrange criterion that takes out duplicate duplicates of identical demography. In any case, the same old ABE frameworks do not strengthen at ease deduplication that makes them costly to be related in a few current stockpiling administrations. Amid this paper, we have got a tendency to show off a novel way to address a function primarily build upon stockpiling infrastructure hold up on relaxed deduplication. The ability infrastructure is made below cross breed cloud style, wherein a non-public cloud administrates the computation and an open cloud provide with the capability. The nonopen cloud is given a trapdoor key related to the coincide cipher text, therewith it will transfer the cipher text over one approach policy into cipher texts of a similar plaintext below the opposite access policies whereas not being responsive to the fundamental plaintext. once getting a storage call, private cloud initial validates the authenticity of the uploaded data based on connected evidence. In case the evidence is valid, the personal cloud executes a tag test formula which anticipate the equivalent data underlying the cipher text. If so, whenever it's a necessity, it reproduces the cipher text into a cipher text of a similar plaintext over an access plan that's that the union set of every access plan. The projected storage system delight in a pair of major glorified. Firstly, it is typically utilizing the privileged publish information with various customers by identifying an access policy instead of providing cryptography key. Secondly, it succeeds with quality idea for syntactic safety whereas existing deduplication plans only reach it below a fragile security plan.

ISSN: 2582-337X

Submitted: 04.12.2020

Accepted: 30.12.2020

Published: 06.01.2021 
Journal of Ubiquitous Computing and Communication Technologies (UCCT) (2020)

Vol.02/ No.04

Pages: 214-218

https://www.irojournals.com/jucct/

DOI: https://doi.org/10.36548/jucct.2020.4.004

\section{REFERENCES}

[1] D. Quick, B. Martini, and K. R. Choo, Cloud Storage Forensics. Syngress Publishing / Elsevier, 2014. [Online]. Available: http://www.elsevier.com/books/cloud-storageforensics/ quick/978-0-12-419970-5

[2] K. R. Choo, J. Domingo-Ferrer, and L. Zhang, Cloud cryptography: Theory, practice and future research directions,Future Generation Comp. Syst., vol. 62, pp. 51-53, 2016.

[3] K. R. Choo, M. Herman, M. Iorga, and B. Martini, Cloud forensics: State-of-the-art and future directions, Digital Investigation, vol. 18, pp. 77-78, 2016.

[4] Y. Yang, H. Zhu, H. Lu, J.Weng, Y. Zhang, and K. R. Choo, Cloud based data sharing with fine-grained proxy reencryption, Pervasive and Mobile Computing, vol. 28, pp. 122-134, 2016.

[5] D. Quick and K. R. Choo, Google drive: Forensic analysis of data remnants,J. Network and Computer Applications, vol. 40, pp. 179- 193, 2014.

[6] A. Sahai and B. Waters, Fuzzy identity-based encryption,in Advances in Cryptology - EUROCRYPT 2005, 24th Annual International Conference on the Theory and Applications of Cryptographic Techniques, Aarhus, Denmark, May 22-26, 2005, Proceedings, ser. Lecture Notes in Computer Science, vol. 3494. Springer, 2005, pp. 457-473.

[7] B. Zhu, K. Li, and R. H. Patterson,Avoiding the disk bottleneck in the data domain deduplication file system, in 6th USENIX Conference on File and Storage Technologies, FAST 2008, February 26- 29, 2008, San Jose, CA, USA. USENIX, 2008, pp. 269-282.

[8] M. Bellare, S. Keelveedhi, and T. Ristenpart,Message-locked encryption and secure deduplication, in Advances in Cryptology - EUROCRYPT 2013, 32nd Annual International Conference on the Theory and Applications of Cryptographic Techniques, Athens, Greece,

May 26-30, 2013. Proceedings, ser. Lecture Notes in Computer Science, vol. 7881. Springer, 2013, pp. 296-312.

[9] M. Abadi, D. Boneh, I. Mironov, A. Raghunathan, and G. Segev, Message-locked encryption for lock-dependent messages, in Advances in Cryptology - CRYPTO 2013 - 33rd Annual Cryptology Conference, Santa Barbara, CA, USA, August 18-22, 2013. Proceedings, Part I, ser. Lecture Notes in Computer Science, vol. 8042. Springer, 2013, pp. 374-391.

[10] S. Keelveedhi, M. Bellare, and T. Ristenpart,Dupless: Serveraided encryption for deduplicated storage, in Proceedings of the 22th USENIX Security Symposium, Washington, DC, USA, August 14-16, 2013. USENIX Association, 2013, pp. 179-194.

[11] S. Bugiel, S. N" urnberger, A. Sadeghi, and T. Schneider,Twin clouds: Secure cloud computing with low latency - (full version),in Communications and Multimedia Security, 12th IFIP TC 6 / TC 11 International Conference, CMS 2011, Ghent, Belgium,

ISSN: 2582-337X

Submitted: 04.12.2020

Accepted: 30.12.2020

Published: 06.01.2021 